EPJ Web of Conferences 73, 04016 (2014)

DOI: $10.1051 /$ epjconf/20147304016

(C) Owned by the authors, published by EDP Sciences, 2014

\title{
Complex-mass scheme and effective field theory
}

\author{
Jambul Gegelia ${ }^{1,2, a}$ \\ ${ }^{1}$ Institut für Theoretische Physik II, Ruhr-Universität Bochum, 44780 Bochum, Germany \\ ${ }^{2}$ Tbilisi State University, 0186 Tbilisi, Georgia
}

\begin{abstract}
The complex-mass scheme applied to hadronic effective field theory is briefly introduced. Some conceptual issues and the application to the calculation of the one-loop corrections to the $\rho$-meson magnetic moment are discussed.
\end{abstract}

\section{Introduction}

Chiral effective field theory (EFT) of the strong interaction describes the $S$-matrix of quantum chromodynamics (QCD) in terms of hadronic degrees of freedom. Hadronic resonances are manifested as poles of the $S$-matrix of QCD. They are represented by corresponding dynamical fields in chiral EFT. Hadronic EFT with resonances is a quantum field theory for unstable particles. Renormalization is a nontrivial issue in the baryonic sector of chiral EFT. Modified version of the on-mass-shell renomalization scheme proved to be very useful in EFT of baryons and mesons, the baryon chiral perturbation theory [1-3]. The complex-mass scheme (CMS) is a generalization of the on-mass-shell scheme to unstable particles [4-6]. Within this scheme the renormalized masses of unstable particles are chosen as poles of dressed propagators. Renormalized masses are included in propagators and the counterterms are treated perturbatively. Applications of the CMS in hadronic EFT can be found in Refs. [7-11]. Here we discuss a couple of conceptual issues and the application in the calculation of the magnetic moment of the $\rho$-meson.

\section{Some conceptual issues}

In CMS the undressed propagator of an unstable particle has a complex pole, therefore one might wonder if the $S$-matrix has a complex pole on the first Riemann sheet. To have closer look at this issue let us consider diagrams contributing to the pion-nucleon scattering in the Roper resonance region in an EFT of the Roper resonance interacting with pions and nucleons, shown in Fig. 1. The first diagram is the leading contribution. The third diagram cancels double pole in the second diagram on the second Riemann sheet. A double pole remains on the physical sheet. Note however that this pole is far away from the applicability of perturbation theory. In that region where this pole appears one needs to re-sum an infinite number of self-energy insertions. Doing so one obtains the dressed propagator of the Roper

\footnotetext{
${ }^{a}$ e-mail: Jambul.Gegelia@tp2.ruhr-uni-bochum.de
}

This is an Open Access article distributed under the terms of the Creative Commons Attribution License 4.0, which permits unrestricted use, distribution, and reproduction in any medium, provided the original work is properly cited. 

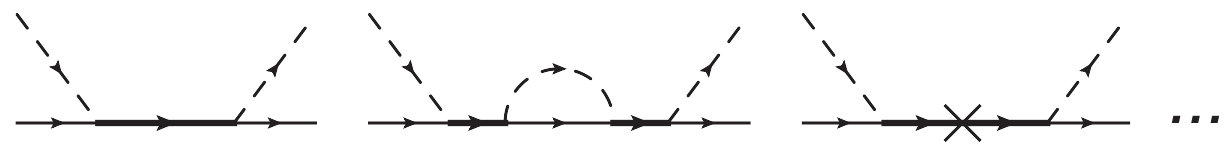

Figure 1. Diagrams contributing to the pion-nucleon scattering in the resonance region. Dashed, solid and bold solid line correspond to the pion, nucleon and the Roper resonance, respectively.

\section{Complex s plane}

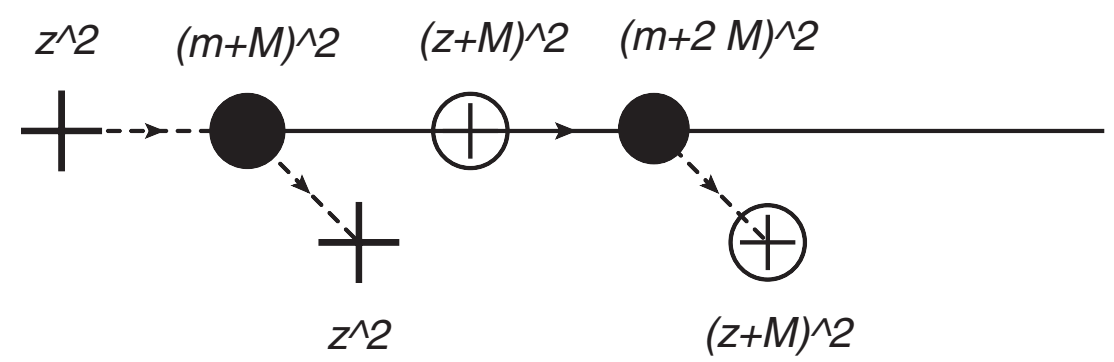

Figure 2. Schematic picture of poles and branch points moving to the complex region due to the change of parameters of the Lagrangian and the Roper resonance becoming unstable. Dashed line indicate the trajectories of the pole and the branch point.

resonance

$$
i S_{R}(p)=\frac{i}{\not p-z_{\chi}-\Sigma_{R}(\not p)},
$$

where $\Sigma_{R}$ stands for the self-energy of the Roper resonance. The dressed propagator of Eq. (1) has a pole only on the second Riemann sheet.

One of the characteristic features of the CMS is the complex branch points of the $S$-matrix explicitly seen in perturbative calculations. This property of the full physical $S$-matrix does not occur in perturtbative calculations by using e.g. the minimal subtraction scheme. By changing the parameters of the Lagrangian the stable particles present in the spectrum of the theory can become unstable and vise-versa. For stable Roper resonance the $\pi N$ scattering amplitude has the corresponding pole in the un-physical region of the physical Riemann sheet at $s=z^{2}$ and corresponding branching point is located at $s=(z+M)^{2}$, where $M$ is the pion mass. By changing the parameters of the Lagrangian the pole and the branch point move to the complex region in the second Riemann sheet as shown in Fig. 2. This property of the full $S$-matrix is manifest within the CMS.

\section{Magnetic moment of the $\rho$-meson}

Below we briefly discuss the application of the CMS to the one-loop order calculation of the magnetic moment of the $\rho$-meson. The explicit form of the corresponding effective Lagrangian and the details of the calculation can be found in Ref. [11].

To define the magnetic moment of the $\rho$-meson, we follow Ref. [12] and consider a process in which the $\gamma \rho \rho$ contributes as a sub-diagram. For definiteness we take $\pi \pi \rightarrow \gamma \pi \pi$, shown in Fig. 3, and parameterize the corresponding amplitude as:

$$
\mathcal{M}=\mathcal{M}_{1}^{\alpha}(-i) D_{\alpha \mu}\left(p_{i}\right) V^{\lambda \mu \nu}\left(q, p_{f}, p_{i}\right)(-i) D_{\nu \beta}\left(p_{f}\right) \mathcal{M}_{2}^{\beta}+\text { Rest },
$$




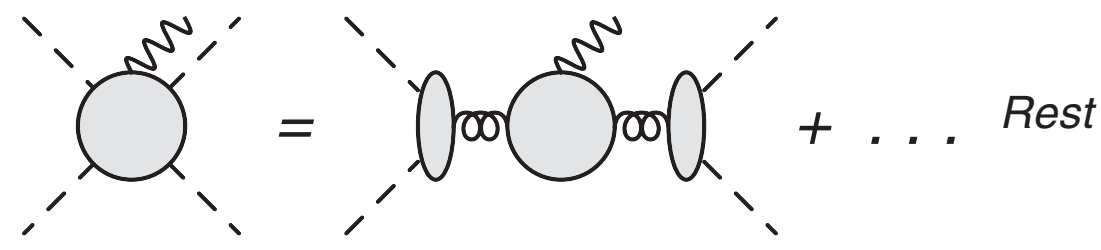

Figure 3. Graphical representation of the $\pi \pi \rightarrow \gamma \pi \pi$ process containing $\gamma \rho \rho$ vertex as a sub-diagram. "Rest" indicates the non-resonant part.

where $\mathcal{M}_{1}^{\alpha}$ and $\mathcal{M}_{2}^{\beta}$ are the $\rho \pi \pi$ vertex functions and $D^{\mu \nu}(p)$ is the dressed propagator of the vector meson. The $\gamma \rho \rho$ vertex function $V^{\lambda \mu \nu}$ can be written as

$$
V^{\lambda \mu \nu}\left(q, p_{f}, p_{i}\right)=\sum_{j} t_{j}^{\lambda \mu v} V_{j}\left(q^{2}, p_{f}^{2}, p_{i}^{2}\right)
$$

where $t_{j}^{\lambda \mu \nu}$ denote the possible tensor structures and $V_{i}\left(q^{2}, p_{f}^{2}, p_{i}^{2}\right)$ are scalar functions. Expanding these coefficient functions about the $z$-pole we define the "on-mass-shell" vertex function

$$
i \Gamma^{\lambda \mu v}\left(q, p_{i}, p_{f}\right)=\sqrt{Z_{V}} \sum_{j} t_{j}^{\lambda \mu v} V_{j}\left(q^{2}, z, z\right) \sqrt{Z_{V}},
$$

where $Z_{V}$ is the (complex) residue of the dressed propagator at the pole $z$. Next we parameterize $\Gamma$ in terms of form factors:

$$
\Gamma^{\lambda \mu \nu}\left(q, p_{i}, p_{f}\right)=f_{1}\left(q^{2}\right)\left(p_{i}^{\lambda}+p_{f}^{\lambda}\right) g^{\mu \nu}+f_{2}\left(q^{2}\right)\left(q^{\nu} g^{\lambda \mu}-q^{\mu} g^{\lambda \nu}\right)+\cdots,
$$

where the ellipsis refer to further structures.

The electric charge $e$ and the magnetic moment $\mu_{\rho}$ are defined in terms of corresponding form factors as

$$
f_{1}(0)=-e, \quad f_{2}(0)=-2 M_{\rho} \mu_{\rho} .
$$

There are tree-level and loop contributions to these quantities. The tree order result reads:

$$
f_{1}^{\text {tree }}(0)=-e, \quad f_{2}^{\text {tree }}(0)=-2 e .
$$

This tree order value of the magnetic moment is in agreement with the findings of Refs. [13, 14].

One-loop diagrams contributing to the $\gamma \rho \rho$ vertex function are suppressed by powers of $\xi=$ $g_{i}^{2} /\left(16 \pi^{2}\right)$ (here $g_{i}$ stand for various coupling constants). Note that even for sizeable values of the coupling $g_{\rho \pi \pi} \sim 6$, this expansion parameter is small $\xi \sim 0.2$.

Strong correction to the charge $f_{1}^{\text {loop }}(0)$ vanishes (only) when the complex wave function renormalization constant, defined as the residue at the complex pole of the dressed propagator is used. On the other hand, one-loop diagrams give a non-vanishing contribution to the magnetic moment. Numerical estimation gives:

$$
f_{2}^{\text {loop }}(0) / e \approx 0.2416-0.0423 i
$$

Thus the one-loop contribution to the magnetic moment is clearly suppressed. This result is to be compared to: $g_{\rho}=2.1 \pm 0.5$ extracted from the $e^{+} e^{-} \rightarrow \pi^{+} \pi^{-} 2 \pi^{0} \mathrm{BaBar}$ data in Ref. [15], and the lattice QCD results, $\left(g_{\rho}\right)_{\text {quenched }} \sim 2.3$ of Ref. [16] and $\left(g_{\rho}\right)_{\text {unquenched }}=1.6(1)$ of Ref. [17]. 


\section{Summary}

The complex-mass scheme is a natural generalization of the on-mass-shell renormalization scheme to processes with unstable particles. It can be applied in the framework of chiral EFT of the strong interaction with explicit degrees of freedom corresponding to unstable particles - resonances. Here we briefly discussed some conceptual issues and the application to the calculation of the $\rho$-meson magnetic moment. At leading order the magnetic moment of the $\rho$-meson is real and the gyromagnetic ratio $g_{\rho}=2$. At one-loop order, the magnetic moment picks up an imaginary part. Corrections to $g_{\rho}$ are of the order of $10 \%$, and the imaginary part is about 0.04 . This result is in reasonable agreement with recent lattice QCD determinations and the result of the data analysis.

This work was supported in part by Georgian Shota Rustaveli National Science Foundation (grant 11/31), and by DFG (SFB/TR 16, "Subnuclear Structure of Matter").

\section{References}

[1] J. Gegelia and G. Japaridze, Phys. Rev. D 60, 114038 (1999)

[2] J. Gegelia, G. Japaridze and X.Q. Wang, J. Phys. G 29, 2303 (2003)

[3] T. Fuchs, J. Gegelia, G. Japaridze and S. Scherer, Phys. Rev. D 68, 056005 (2003)

[4] R. Stuart, in $Z^{0}$ Physics, ed. J. Tran Thanh Van (Editions Frontiers, Gif-sur-Yvette, 1990), p. 41

[5] A. Denner, S. Dittmaier, M. Roth and D. Wackeroth, Nucl. Phys. B 560, 33 (1999)

[6] A. Denner, S. Dittmaier, M. Roth and L.H. Wieders, Nucl. Phys. B 724, 247 (2005)

[7] D. Djukanovic, J. Gegelia and S. Scherer, Phys. Lett. B 690, 123 (2010)

[8] D. Djukanovic, J. Gegelia, A. Keller and S. Scherer, Phys. Lett. B 680, 235 (2009)

[9] T. Bauer, J. Gegelia and S. Scherer, Phys. Lett. B 715, 234 (2012)

[10] T. Bauer, D. Djukanovic, J. Gegelia, S. Scherer and L. Tiator, AIP Conf. Proc. 1432, 269 (2012)

[11] D. Djukanovic, E. Epelbaum, J. Gegelia and U.-G. Meißner, arXiv:1309.3991 [hep-ph]

[12] J. Gegelia and S. Scherer, Eur. Phys. J. A 44, 425 (2010)

[13] D. Djukanovic, M.R. Schindler, J. Gegelia, and S. Scherer, Phys. Rev. Lett. 95, 012001 (2005)

[14] C. Lorce, Phys. Rev. D 79, 113011 (2009)

[15] D. García Gudiño and G. Toledo Sánchez, arXiv:1305.6345 [hep-ph]

[16] J.N. Hedditch, W. Kamleh, B.G. Lasscock, D.B. Leinweber, A.G. Williams and J.M. Zanotti, Phys. Rev. D 75, 094504 (2007)

[17] M. Gurtler et al. [QCDSF Collaboration], PoS LATTICE 2008, 051 (2008) 